Maximal JSW reduction (one knee) significantly correlated with entry and final COMP levels $(\rho=0.201, \mathrm{p}<0.05, \rho=$ $0.187, \mathrm{p}<0.05)$; JSW reduction from both knees correlated with final COMP level $(\rho=0.162, \mathrm{p}<0.05)$; the correlation with entry COMP level nearly reached the significance level $(\mathrm{p}$ $=0.07)$. Chondrex levels did not correlate with JSW reduction at all.

Conclusion COMP could represent biochemical predictor of radiographic progression in knee OA. The possible predictive role of chondrex in radiographic progression of knee OA was not proven in this study.

\section{SAT0087 THE EFFECT OF MELOXICAM VERSUS USUAL CARE NSAIDS FOR THE TREATMENT OF OSTEOARTHRITIS IN A USUSAL CARE SETTING. THE RESULTS OF THE IMPROVE TRIAL}

${ }^{1} \mathrm{P}$ Gagnier, ${ }^{2} \mathrm{G}$ Singh, ${ }^{3} \|$ Reed, ${ }^{1} \mathrm{M}$ Kobe, ${ }^{1} \mathrm{P}$ Arnold, ${ }^{4} \mathrm{E}$ Hwang. ${ }^{1}$ Clinical Research; ${ }^{2}$ Medicine/lmmunology and Rheumatology, Stanford University Medical Center, Palo Alto, CA, USA; ${ }^{3}$ Rheumatology, Fallon Clinic, Worcester, MA, USA; ${ }^{4}$ Biometrics and Data Management, Boehringer Ingelheim Pharmaceuticals, Inc., Ridgefield

10.1136/annrheumdis-2001.462

Background Traditionally, NSAIDs have been the mainstays for managing symptoms of osteoarthritis (OA). While NSAIDs generally are considered to be effective in managing pain and inflammation, they frequently are associated with gastrointestinal side effects or lose their effectiveness leading to discontinuation of therapy or switching to another NSAID. These side effects can add significantly to the direct medical cost of managing the OA patient population. This suggests that there is dissatisfaction with current therapies on the part of the patient, physician or both. The time to discontinuation or change to another NSAID may be used as one measure that can examine the interrelated reasons for discontinuation, mainly efficacy and tolerability. Currently, there are no published randomised prospective studies that have examined the time to NSAID discontinuation; or NSAID switches and

Objectives The objective of this study was to compare the percentage of treatment successes or failures in patients randomised to meloxicam $7.5 \mathrm{mg}$ vs. usual care prescription NSAIDS in a variety of managed care settings (MCOs). A success was defined as a patient who started on their initially prescribed study NSAID and never received a different prescription NSAID during the trial. During the trial, the patient could have temporarily discontinued the new study prescription NSAID, restart the same drug and be considered a treatment success. A treatment failure was defined as a patient who started on an originally prescribed study NSAID and changed to another NSAID at any point during the trial.

Methods This was a multicenter, randomised, parallel-group, open-label trial of meloxicam $7.5 \mathrm{mg}$ versus usual care administration of prescription formulary NSAIDs for the treatment of patients with OA in a variety of MCO models. Patients with OA of the hip, knee, hand or spine were randomised to receive either meloxicam $7.5 \mathrm{mg}$ or any other prescription NSAID that they had not previously been treated with for their OA based on the patients formulary. The study was designed to mirror actual MCO practices and not add costly and unnecessary study-driven procedures at enrolment and throughout the observation period. The treatment/observation period was 6 months in duration and assessments were made to determine the reasons for permanent discontinuation from the initial prescription NSAIDs, number of
NSAID therapies, as well as the health care utilisation, efficacy and safety.

Results There were 1,321 patients entered into the trial and approximately $67 \%$ of the patients were female and $95 \%$ Caucasian. The mean age was approximately 64 years and the mean duration of OA was over 9 years. Rates of NSAID switching and efficacy will be discussed.

Conclusion The study was well accepted by physicians and patients.

\section{SAT0088 COST-BENEFIT ANALYSIS OF GLUCOSAMINE SULFATE USE}

${ }^{1} \mathrm{LG}$ Mantovani, ${ }^{1} \mathrm{~A}$ Belisari, ${ }^{2} \mathrm{C}$ Cristoforetti, ${ }^{2} \mathrm{G}$ Giacovelli, ${ }^{2} \mathrm{LC}$ Rovati. ${ }^{1}$ Centre of Pharmacoeconomy, University of Milan, Italy; ${ }^{2}$ Clinical Pharmacology Department, Rotta Research Lab. S.p. A., Monza Milan, Italy

\subsection{6/annrheumdis-2001.463}

Background Osteoarthritis (OA) is a disabling and costly disease (see Belisari's and Mantovani's abstract). Glucosamine sulfate has shown to be effective in reducing the severity of OA as measured through the Lequesne index (LI).

Objectives To evaluate the the pharmacoeconomic profile of Glucosamine sulfate in Italy.

Methods Cost-benefit analysis of glucosamine sulfate use in the context of the Italian society. We compared 2 different pharmacological interventions in OA patients: piroxicam vs. glucosamine sulfate. We considered direct medical costs (e.g. treatment), direct non-medical (e.g. home adaptation, transport, etc.) and indirect (e.g. wage losses) costs. Costs are expressed in Euros 2000. Benefits were measured on the results of a reference randomised clinical trial. They were expressed in terms of LI score reduction, and were turned into monetary terms using the equation estimated in the study by Belisari and Mantovani, which shows a significant association between severity of the disease and costs (higher costs in more severe patients).

Results Although glucosamine sulfate therapy cost is higher than piroxicam one (81 vs. 33 Euros, the intervention with glucosamine sulfate resulted in a potential net saving of almost 11 Euro/patient in 90 days and 110 Euros/patient in 150 days attributable to its higher efficacy.

Conclusion Based on our estimates, glucosamine sulfate has a favourable cost benefit profile compared to piroxicam.

\section{REFERENCE}

1 Rovati LC. Osteoarthritis Cartilage 1997;5(Suppl A):72

\section{SAT0089 ROFECOXIB PROVIDES SUPERIOR RELIEF OF SYMPTOMS OF OSTEOARTHRITIS (OA) COMPARED TO CELECOXIB}

${ }^{1} T J$ Schnitzer, ${ }^{2} \mathrm{AJ}$ Kivitz, ${ }^{3} \mathrm{M}$ Greenwald, ${ }^{4} \mathrm{RM}$ Fleischmann, ${ }^{5} \mathrm{D}$ Matzura-Wolfe, ${ }^{5} \mathrm{AB}$ Polis, ${ }^{5} \mathrm{ME}$ Dixon, ${ }^{5} \mathrm{TW}$ Dobbins, ${ }^{5} \mathrm{GP}$ Geba. ${ }^{1}$ Northwestern University, Chicago IL, USA; ${ }^{2}$ Altoona Center for Clinical Research, Ducansville, PA, USA; ${ }^{3}$ Advances in Medicine, Rancho Mirage, CA, USA; ${ }^{4}$ Metroplex Clinical Research, Dallas, TX, USA; ${ }^{5}$ Merck and Co., Inc., West Point $P A, U S A$

\subsection{6/annrheumdis-2001.464}

\section{Background}

Objectives We performed a randomised, double-blind, clinical trial to evaluate the efficacy and safety of rofecoxib and celecoxib at highest indicated once daily doses in osteoarthritis. 
Methods 1082 patients meeting entry criteria for OA, responsive to NSAIDs, were randomised 3:3:1 to treatment with rofecoxib $25 \mathrm{mg}$ qd $(\mathrm{n}=471)$, celecoxib $200 \mathrm{mg}$ qd $(\mathrm{n}=460)$ or placebo $(\mathrm{n}=151)$. Efficacy was assessed over the first 6 days of therapy and at weeks 2, 4, and 6 by WOMAC questionnaire and patient global assessment of response to therapy (PGART).

Results Demographics were well balanced. Significantly more patients on placebo discontinued prematurely compared to both active groups ( $p<0.001)$ mainly due to lack of efficacy. Rofecoxib provided statistically superior relief of night pain $(\mathrm{p}=$ $0.023)$, morning stiffness $(\mathrm{p}=0.002)$, rest pain $(\mathrm{p}=0.023)$, and walking pain $(\mathrm{p}=0.005)$ compared to celecoxib. Rofecoxib was significantly superior to celecoxib on all WOMAC subsacles, including pain $(\mathrm{p}=0.008)$, stiffness $(\mathrm{p}=0.001)$ and physical function $(\mathrm{p}=0.01)$. Rofecoxib was superior to celecoxib in $\%$ of patients with good or excellent PGART over 6 weeks $(\mathrm{p}=$ 0.014 ) and provided quicker onset of efficacy as assessed by time to first report of good or excellent response $(p<0.001)$. Both active groups were superior to placebo on efficacy endpoints. Incidence of clinical AEs, drug related AEs, serious AEs, and discontinuations due to AEs was similar between active groups.

Conclusion In this study, once daily doses of rofecoxib provided superior relief of pain and other related symptoms in OA compared to celecoxib and placebo. All treatments were generally well tolerated.

\section{SAT0090 CORRELATION BETWEEN PROXIMAL FEMUR BONE MINERAL DENSITY AND HIP OSTEOARTHRITIS: PRELIMINARY DATA}

N Malavolta, P Mastrapasqua, L Lisi, M Frigato, MG Prandin, S Gnudi. Medicina Interna E dell'Invecchiamento, Azienda Ospedaliera Di Bologna-Policlinico S. Orsola -Malpighi, Bologna, Italy

\subsection{6/annrheumdis-2001.465}

Background Osteoarthritis (OA) is a very frequent disease in postmenopausal women. The question whether high bone mineral density (BMD) plays a role in the development of this disease is under investigation.

Objectives Here we report the preliminary data of a study aimed at investigating the relationship between the BMD of healthy women and women with OA of the hip.

Methods Exclusion criteria included all diseases and drugs known to cause BMD loss. Till now 136 age matched women have entered the study: 46 with OA (mean age 65.76 , SD 8.07) and 92 healthy individuals (mean age 65.60, SD 8.12). All subjects underwent Dual Energy X-ray Absorptiometry (DEXA-XR 36 Norland) measurements of the femoral neck, trochanter, Ward?s and the lumbar spine (L2-L4). Age, age of menopause, height and weight were also collected. Data were analysed using the Anova test to compare groups. The ability of selected parameters to discriminate the two groups of patients was tested by logistic regression.

Results Weight $(p=0.003)$ and femoral neck BMD ( $p=0.045)$ were significantly higher in women with OA than in healthy women. None of the other variables were significantly different in the two groups. At the logistic regression both age corrected weight (OR: 1.05 ; 95\% CI: 1.01-1.09) and age corrected femoral neck BMD (OR: 1.01; 95\% CI: 1.00-1.03) were able to significantly discriminate the two groups of women. Both variables correctly classified $13.04 \%$ of OA while $93.48 \%$ of healthy women were correctly classified by the variable weight and 97.83\% the femoral neck BMD.

Conclusion Our data suggest that the body weight and femoral neck BMD are the main risk factors for OA of the hip. The fact that at the trochanter and at the Ward?s (where BMD measurement does not discriminate between the two groups) the cortical component of bone is lower than at the femoral neck should indicate that high cortical bone density is a marker of OA.

\section{SAT0091 EFFECTS OF JOINT LAVAGE AND HYALURONIC ACID INFILTRATION IN PATIENTS WITH OSTEOARTHRITIS OF THE KNEE}

MA Caracuel, MC Muñoz-Villanueva, A Escudero, R Veroz, G Frias, J Vacas, C Perez, M Romero, V Perez, J Gonzalez, FG Martinez, E Collantes. Rheumatology Service, Hospital Universitario Reina Sofia, Cordoba, Spain

\subsection{6/annrheumdis-2001.466}

Background Infiltration with hyaluronic acid (HA) and joint lavage (JL) have recently been proposed as local treatments for osteoarthritis of the knee. Joint lavage is a therapeutic treatment involving passing cold sterile $0.9 \%$ saline through the knee joint in order to have the liquid reach the inside of the joint capsule. Objectives To evaluate the efficacy of joint lavage and intraarticular infiltration with hyaluronic acid, alone and in combination (JLHA), as symptomatic treatments for knee osteoarthritis.

Methods An overall 37 patients (8 males and 29 females) aged 43-85 years with knee osteoarthritis of radiological grade II on the Kellgren scale were randomly classified into three thera-peutic groups, namely: JL $(\mathrm{n}=14)$, HA $(\mathrm{n}=10)$ and JLHA $(\mathrm{n}=$ 13). The patients in group $\mathrm{JL}$ received joint lavage, on day 0 only; those in group JLHA were subjected to joint lavage on day 0 and to infiltration on days $0,7,14,21$ and 28. Outcome measures evaluated at baseline, at days 7 and 30 included severity of pain (VAS), Global status (VAS) and Lequesne?s functional index.

Results The results of the Global Status difference from baseline are summarised in the following Table 1:

\begin{tabular}{lll} 
Abstract & SAT0091 Table 1 & \\
\hline & $\begin{array}{l}\text { Assessment at week } \\
\text { Differential Global Status } \\
\text { (significance level) }\end{array}$ & $\begin{array}{l}\text { Assessment at month } \\
\text { Differential Global Status } \\
\text { (significance level) }\end{array}$ \\
\hline JL vs HA & -5.14 vs $-3.10(p=0.03)$ & -5.07 vs $-2.80(p=0.02)$ \\
HA vs & -3.10 vs $-5.38(p=0.01)$ & -2.80 vs $-5.76(p=0.02)$ \\
JLHA & & -5.07 vs $-5.76(p=0.36)$ \\
JL vs & -5.14 vs $-5.38(p=0.59)$ & \\
JLHA & &
\end{tabular}

Conclusion Our results suggest significant differences between the tested treatments, Joint lavage alone and followed by infiltration with the hyaluronic acid is more effective than local infiltration with hyaluronic acid alone. 